\title{
High-precision digital audio waveform synthesis using a multi-frequency interpolation technique
}

\author{
Patrick Gaydecki \\ School of Electrical and Electronic Engineering, University of Manchester, Manchester, UK \\ E-mail: patrick.gaydecki@manchester.ac.uk
}

\begin{abstract}
A description is given of a digital, real-time, multi-frequency function generator for audio bandwidth applications. A novel feature of the system is the embedded algorithm, which employs a bank of parallel interpolators, rather than a look-up table, to synthesise complex functions with high precision and flexibility. The complete instrumentation comprises a high-level software design tool operating on a conventional desktop or notebook PC and a separate hardware unit incorporating a powerful real-time digital signal processor unit, operating at 275 million multiplication-accumulations per second (MMACs) and a dual channel 24-bit codec with a maximum output rate of 200 kilo-samples per second. Using total harmonic distortion (THD) analysis, the purity of the waveforms has been compared with those generated by traditional analogue instruments; this analysis has confirmed that the new instrument has a consistently superior performance over such devices. Testing established that for a $2 \mathrm{kHz}$ sine wave, for example, there was an order of magnitude improvement in THD performance over a commercially available analogue function generator. The algorithm and associated hardware offer the combined advantages of function flexibility, ease of implementation, accuracy and the unique ability to alter, in real-time, the frequencies, magnitudes and phases of individual harmonics within any given arbitrary waveform. Digital function generation and waveform synthesis represent key components of any DSP course; at the University of Manchester it is taught as a formal discipline through lectures, and from a practical context during our laboratory classes, in which students gain an understanding of digital methods and how they excel over traditional analogue circuits designed for the same purpose.
\end{abstract}

Keywords look-up table; multi-frequency synthesis; real-time interpolation; waveform synthesis

The evolution of high-speed DSP devices has resulted in a wide variety of products intended for signal and function generation; these are operations that have traditionally been performed by analogue circuitry. In fact, the emergence of digitally-based signal generators has enabled certain specific operations to be realised in real time that would have been extremely difficult to implement through analogue means. In particular, arbitrary functions are almost impossible to generate using analogue circuitry, precisely because the signals cannot be described by mathematical functions. When implemented using digital technology, arbitrary functions are as easy to produce as well-known standard waveforms such as sine, square, triangle and ramp (saw tooth) waveforms. Here, we describe a flexible DSP-based unit that is capable of generating real-time waveforms using a novel parallel bank interpolation technique for synthesis of sine, multi-frequency and modulated sine waveforms. The interpolation algorithm, which is straightforward to realise, enables such functions to be generated with very high frequency resolution and, moreover, permits frequencies to be altered in real-time via interaction with the user through appropriate control software. 
From an educational perspective, interpolation is not only appropriate in any course that teaches foundations of DSP, it clearly has links to linear algebra, curve fitting and signal synthesis in general. This is significant, since there is sometimes a perceived disconnect between subjects taught at a theoretical level and practical or engineering problems. With this case in particular, the associations are made unambiguously through a series of structured laboratory exercises.

Although the look-up table represents a simple and intuitive approach to real-time function generation and is widely used, ${ }^{1-4}$ it has both merits and limitations. One clearly useful feature is its flexibility; a computer can be used to pre-compute arbitrary functions, the values of which may then be stored and played out in real time as required. However, the length of the function is restricted by the amount of memory available. Additionally, there is a more fundamental constraint, which concerns the accuracy with which sine waves may be generated at specific frequencies. ${ }^{5}$ Consider a sine wave that has been synthesised over a single cycle, with 8 equispaced points in the look-up table. If the digital-to-analogue conversion rate is $1 \mathrm{kHz}$, then the sine wave frequency generated will be $125 \mathrm{~Hz}$. Now, in order to generate an accurate sine wave, the values held in the look-up table must be such that they end and start with no discontinuities, i.e. typically they must be lie on the zero-crossing points. If this is not done, waveform distortion will occur. Hence, if only a single cycle is used in the computation process (as in this example), the next highest frequency that may be generated, using 7 points, is $142.857 \mathrm{~Hz}$. If better frequency resolution is needed, more cycles must be included in the look up table - i.e. if two cycles are stored, then the next highest frequency that can be generated accurately is $(16 / 15) \times 125=133.33 \mathrm{~Hz}$. This clearly means two things: first, the better the frequency resolution required, the larger the look-up table must be; second; given that an entirely new look up table must be computed for each frequency, the method is not fast enough to permit frequency alteration in real time. There are methods to ameliorate the performance, typically by employing inter-point interpolation in real-time. ${ }^{6}$ This form of interpolation is fundamentally different from the one proposed here, and is usually implemented as a linear piecewise approximation algorithm; this requires the equation of the slope between two adjacent points to be computed each time a sample is generated. This adds to the complexity of the realtime procedure and the linear approximation will, moreover, adversely impact on the spectral purity of the waveform. This basic scheme may be improved by employing series expansions, but at the cost of even greater complexity. The fact remains that the look-up table algorithm fundamentally is circumscribed by its discrete nature and solutions to obviate this are either inelegant or necessitate lengthy algorithms which degrade the speed of the real-time performance.

\section{Real-time function generation using parallel bank interpolation}

The system discussed here exploits interpolation to generate individual sine waves, and for complex multi-frequency signals it employs a bank of parallel interpolators, the outputs of which are summed to produce the final composite waveform. This method is far more accurate than the look-up table approach and provides much

International Journal of Electrical Engineering Education, Volume 50, Number 3 (July 2013), (C Manchester University Press 
greater flexibility and frequency resolution. Compared with the look-up table method, it uses minimal memory resource; specifically, for each sine wave, it requires five 24-bit words to store the polynomial coefficients and a single word to store the time increment. In comparison, a single look-up table may comprise several hundred or thousand values. Since the interpolation system calculates the output in real-time rather than using stored values, it is ideally suited to digital-function generators, in which the frequency may be changed at any point by the user with no perceptible delay. The high-level software first fits a polynomial to approximate the sine wave function over a half a cycle (in fact, only a quarter of a cycle is required but from an implementation perspective it is easier if the above approach is followed). Since the sine wave is a smoothly changing analytic function, it can be approximated extremely accurately using, for example, a $4^{\text {th }}$ order fit. Figure 1 shows two curves: (a) a half-cycle sine wave function and (b) its approximation using a $4^{\text {th }}$ order polynomial fit. (These functions have been normalised to allow the polynomial to be calculated correctly by the fixed-point processor). The $R^{2}$ coefficient of the fit is 0.9999985. This method is so precise that the quality of the real-time function it produces is far better than that generated by expensive analogue instruments, as confirmed by the THD tests described later.

The high-level software employs Gaussian elimination to obtain the polynomial coefficients, a method which is described in many engineering texts. ${ }^{7}$ In brief, this works as follows: consider the solution for a $4^{\text {th }}$ order polynomial thus:

$$
\begin{aligned}
& y_{0}=a_{0} x_{0}^{0}+a_{1} x_{0}^{1}+a_{2} x_{0}^{2}+a_{3} x_{0}^{3}+a_{4} x_{0}^{4} \\
& y_{1}=a_{0} x_{1}^{0}+a_{1} x_{1}^{1}+a_{2} x_{1}^{2}+a_{3} x_{1}^{3}+a_{4} x_{1}^{4} \\
& y_{2}=a_{0} x_{2}^{0}+a_{1} x_{2}^{1}+a_{2} x_{2}^{2}+a_{3} x_{2}^{3}+a_{4} x_{2}^{4} \\
& y_{3}=a_{0} x_{3}^{0}+a_{1} x_{3}^{1}+a_{2} x_{3}^{2}+a_{3} x_{3}^{3}+a_{4} x_{3}^{4} \\
& y_{4}=a_{0} x_{4}^{0}+a_{1} x_{4}^{0}+a_{2} x_{4}^{0}+a_{3} x_{4}^{0}+a_{4} x_{4}^{4}
\end{aligned}
$$

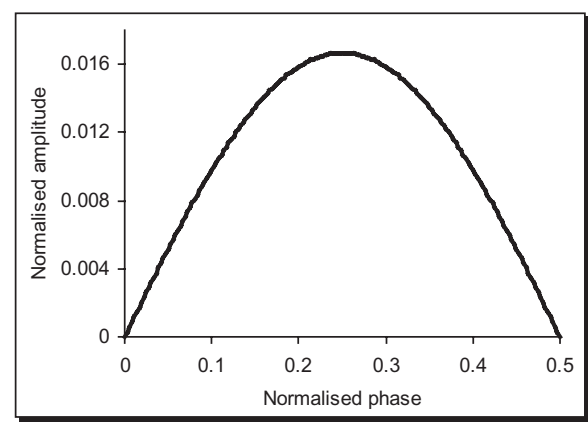

(a)

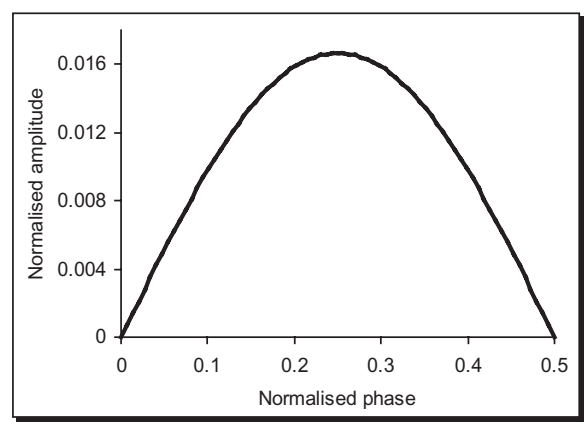

(b)

Fig. 1 (a) Half-cycle sine wave and (b) its approximation using a $4^{\text {th }}$ order polynomial fit. 
Both the $y$-values and $x$-values are known, since these are the vertices. The objective is therefore to find the set of coefficients which will allow the polynomial given by

$$
y=\sum_{m=0}^{4} a_{m} x^{m}
$$

to pass exactly and smoothly through the vertices. In essence, Gaussian elimination involves re-arranging the equation set and cancelling using back-substitution to allow the determination of the coefficients.

The polynomial coefficients are then normalised and downloaded to the DSP system, stored in its memory and evaluated in real time to produce the sine wave. Normalization is necessary because the high-level software uses floating point arithmetic, whereas the real-time processor employs a fixed point, fractional ALU. Hence all coefficients must be scaled and expressed as integers. Regardless of the frequency selected, the coefficients never change - all that changes is the $x$-axis (time) step of the system, which determines the output frequency. To select a different frequency, only a single value is downloaded to the system, i.e. the time step. Figure 2 depicts the real-time sine wave generator interface from the control software. It allows the user to generate two independent sine waves on channels 1 and 2, with a maximum frequency of $22 \mathrm{kHz}$ and a frequency resolution of $0.01 \mathrm{~Hz}$. (In fact, the actual frequency resolution is better than this, $0.00286 \mathrm{~Hz}$, since the DAC is clocked at $48 \mathrm{kHz}$ and the device uses a 24-bit word length. However, for the sake of sensible units the software uses the larger step). The sliders may be adjusted in real-time to alter the sine wave on-the-fly. In addition, the system allows the user to produce multi-frequency signals or modulate one channel by the other. It is clear that there are several advantages of this system over the look-up table method. First, the memory requirement is minimal, requiring 4 words for the coefficients and one word for the time increment. Second, to alter the frequency, only a single new time step

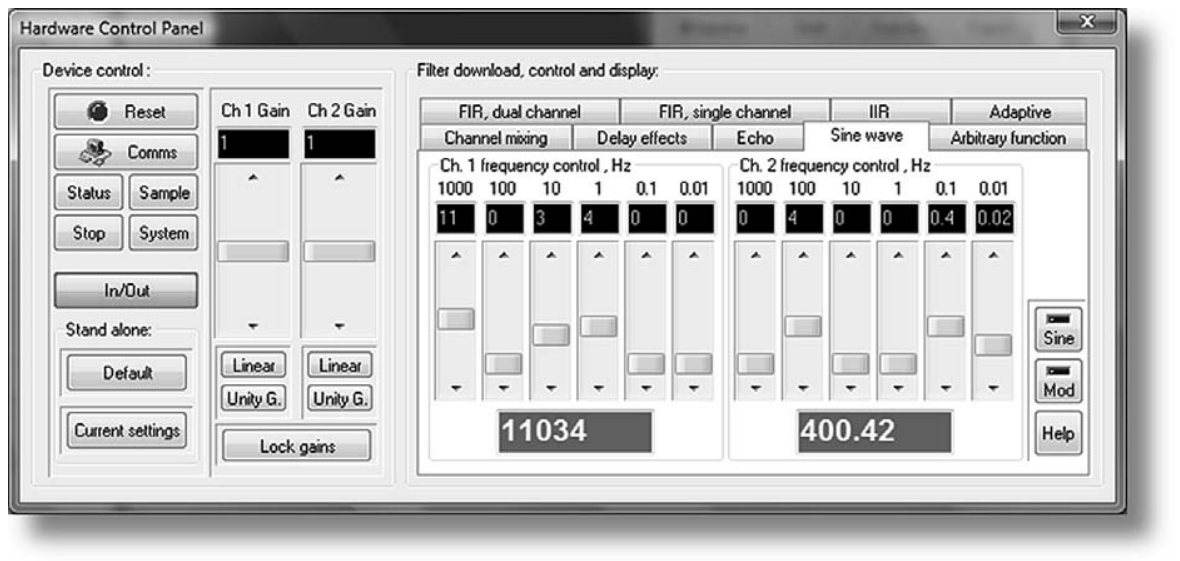

Fig. 2 Real-time sine wave generator interface from the instrument.

International Journal of Electrical Engineering Education, Volume 50, Number 3 (July 2013), (C Manchester University Press 
need be downloaded, so the system is ideally suited for real-time control applications. Finally, this system is not constrained to using an integer number of samples to represent a complete cycle. In fact, the frequency resolution is limited only by the word length of the system; it is quite straightforward for this system to produce a digital sine wave with a frequency step of $3 \mathrm{mHz}$ across the entire frequency range.

\section{Functional description of the real-time firmware}

The polynomial interpolation method is also commonly used to generate transcendental functions. ${ }^{8,9}$ This is possible because a sine wave, for example, has a single inflexion point over half a cycle, which allows low-order fits to be employed. However, the technique is problematic for arbitrary or multi-frequency functions since high order polynomials give rise to unwanted inflexions which will not be representative of the curve required. This issue is resolved here by employing loworder polynomials within a bank of parallel interpolators, whose outputs are fed to a delay system (for phase control), gain adjustment and final summation operation. The entire algorithm is expressed in real-time assembly code and again controlled by high-level software. Figure 3 depicts the flow-chart of the real-time firmware system.

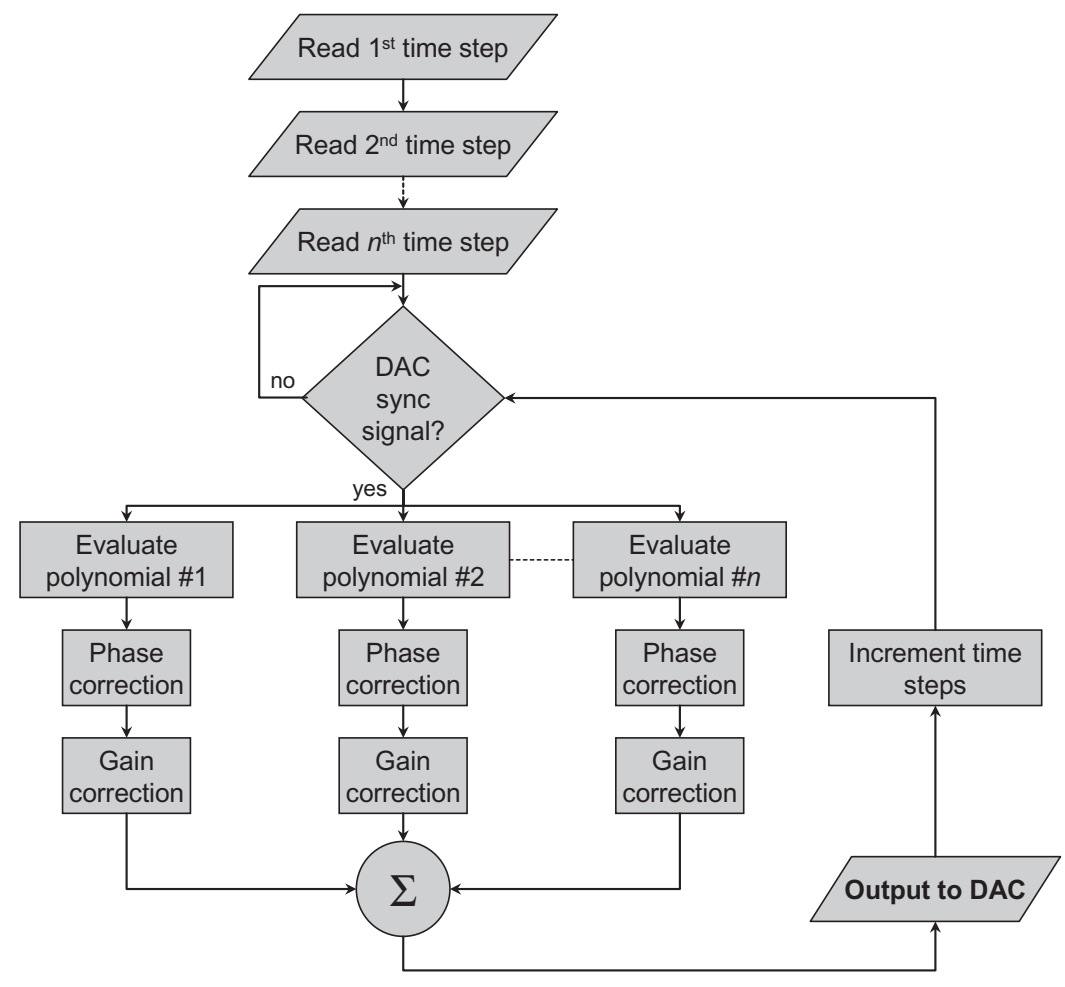

Fig. 3 Flowchart of real-time arbitrary function system.

International Journal of Electrical Engineering Education, Volume 50, Number 3 (July 2013), (C Manchester University Press 
Within the context of multi-frequency signal generation, an important question arises regarding speed of operation and the ability to synthesise elaborate functions. This may be answered straightforwardly enough by considering the power of the DSP unit and the sample rate of the digital-to-analogue converter (DAC). In this case, we are employing a Freescale DSP56321 processor, capable of performing 275 million multiplications and accumulations per second, i.e. 275 MMACs. For typical audio purposes, the DAC will be clocked at 48000 samples per second. Each time the DAC generates a sample, eqn (2) must be evaluated, which requires 5 multiplication-accumulations (MACs). Furthermore, a single MAC is required to implement a phase delay and a final single MAC for the addition applied to the parallel interpolator bank for gain and summation. Hence, the theoretical maximum number of sine waves, $N_{s}$, that may be summed in this way (whilst clocking the DAC at 48000 samples per second), is given by

$$
N_{s}=\frac{275 \times 10^{6}}{48 \times 10^{3} \times 7}=818.45
$$

Although the multiplier architecture is designed to work at $100 \%$ efficiency for processing MAC instructions, in practice there are overheads associated with data output and memory accesses to obtain coefficients. Hence a more realistic figure for $N_{s}$ is approximately 800 . In terms of signal properties, it is clear that very complex functions may be synthesised in this manner, the more so because the magnitude and phase of each individual sine component may be defined precisely.

\section{Hardware description}

The major hardware components of the waveform synthesiser are conceptually very straightforward, and are shown in Fig. 4. A central DSP device, in this case a Freescale DSP56321, operating at 275 million MMACs, is responsible for producing the signal in digital form as described above, with the real-time firmware resident within its high-speed memory. This signal is fed to the DAC section of a Cirrus CS4271 codec, which is a dual-channel device with 24-bit resolution and userselectable sample rates ranging between $4 \mathrm{kHz}$ to $200 \mathrm{kHz}$. The output of the DAC is connected to an anti-aliasing filter and the final analogue signal is thus sent to the outside world. In addition to the DSP and DAC components, the system incorporates memory and a communications interface through which it receives instructions from purpose-written control software operating on a conventional PC running Microsoft Windows XP or Vista.

\section{Performance evaluation}

Total Harmonic Distortion (THD) analysis represents a simple and convenient mechanism for establishing the purity of transcendental functions such as sine waves. If the amplitude of the fundamental frequency is given by $B_{0}$ and the amplitudes of subsequent harmonics by $B_{1}, B_{2}, \ldots B_{n}$, then total harmonic distortion, $D_{t h}$ may be defined as

International Journal of Electrical Engineering Education, Volume 50, Number 3 (July 2013), (C) Manchester University Press 

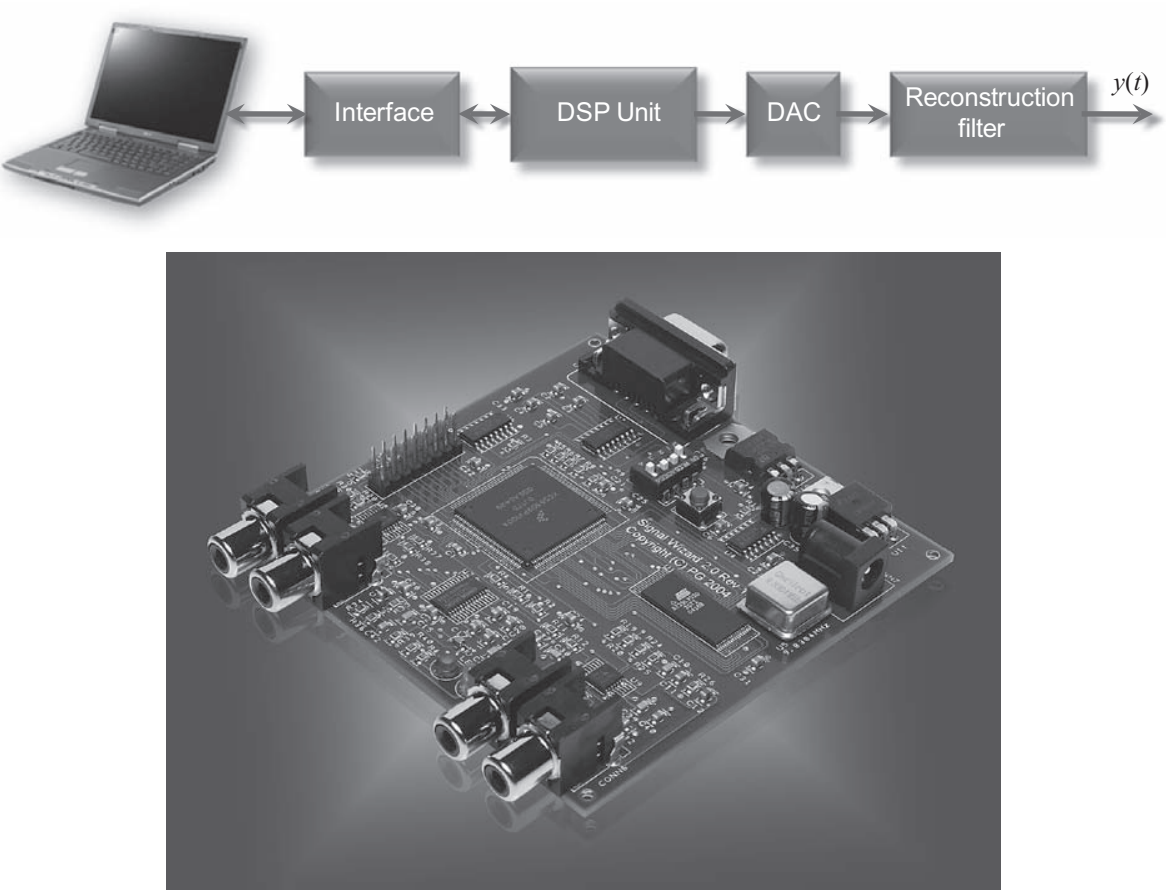

Fig. 4 DSP signal function generator concept (top) and actual hardware (bottom).

TABLE 1 THD figures for digital vs analogue sine wave generation

\begin{tabular}{lccc}
\hline System & \%THD@2 kHz & \%THD@3 kHz & \% THD@5 kHz \\
\hline Digital & 0.057 & 0.084 & 0.334 \\
Analogue & 0.663 & 0.861 & 17.55 \\
\hline
\end{tabular}

$$
D_{t h}=\sqrt{\left(\frac{B_{1}}{B_{0}}\right)^{2}+\left(\frac{B_{2}}{B_{0}}\right)^{2}+\ldots+\left(\frac{B_{n}}{B_{1}}\right)^{2}}
$$

THD measurements were made for several spot frequencies, both for the digital interpolation system and, for comparison, a conventional bench-top analogue function generator (Thurlby Thandar model TG230). In each case, the sine waves were digitised and analysed by a high-precision waveform analyser with 24-bit precision. Table 1 confirms that in every case, the performance of the digital interpolation system was superior to that of the analogue function generator. For illustration purposes, Fig. 5 shows spectra (dB magnitude) from both the digital instrument (a) and the analogue function generator (b), for a $1 \mathrm{kHz}$ sine wave. A cursory examination of the spectra supports the numerical analysis respecting the enhanced performance

International Journal of Electrical Engineering Education, Volume 50, Number 3 (July 2013), (C) Manchester University Press 


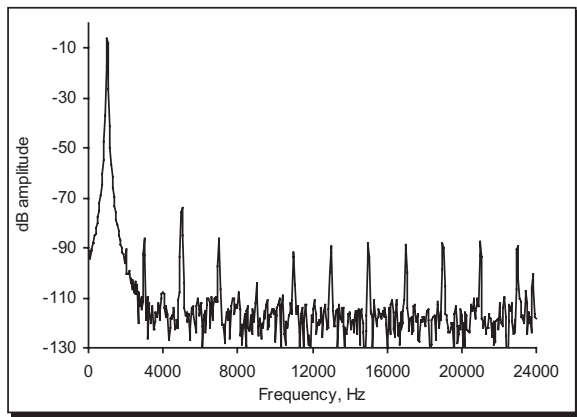

(a)

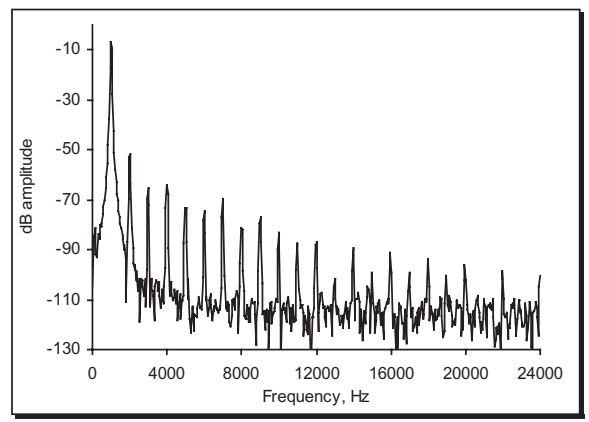

(b)

Fig. 5 Spectra of a $1 k \mathrm{~Hz}$ sine wave produced by (a) the digital interpolation system and (b) the analogue function generator.

of the digital system. Furthermore, because the codec employed in the design is a dual channel device with synchronous outputs, it is possible to generate exact replicas of a sine wave in each channel, locked in time but phase or gain adjusted as required.

In a second test, the system was configured to generate a function which was a composite of four sine waves, at $10 \mathrm{kHz}$ with a gain of $0.071,8 \mathrm{kHz}$ with a gain of $0.048,7 \mathrm{kHz}$ with a gain of 0.143 and $6 \mathrm{kHz}$ with a gain of 0.154 . The output was then fed to the same waveform analyser and examined for spectral purity and noise performance. The signal and spectrum are shown in Fig. 6. For purposes of clarity, the spectrum shows a linear magnitude response and confirms that the composite signal contains only those harmonics as specified in the design. Since each harmonic is computed separately and then summed within the real-time code, it is reasonable to assume that such arbitrary waveforms will exhibit the same spectral purity as their single-frequency counterparts.

\section{Discussion}

Although the look-up table algorithm is more widely applied than the interpolation method for digital function generation (primarily because of its ease of implementation), it is nevertheless inherently limited by its lack of run-time flexibility and the fundamentally discrete nature of its expression. Whilst the method is both accurate and stable, real-time alteration of the output frequency is performed by changing the frequency of the system clock, which is undesirable. In contrast, although interpolation techniques are steadily increasing in popularity, ${ }^{10}$ their widespread adoption has been impeded by the difficulties associated with synthesizing complex functions. Several authors report the use of algorithms which combine the look-up table approach with function approximation methods, ${ }^{11-13}$ but the use of stored coefficients becomes problematic when real-time frequency manipulation is required. The method described here addresses these problems successfully by exploiting a bank 


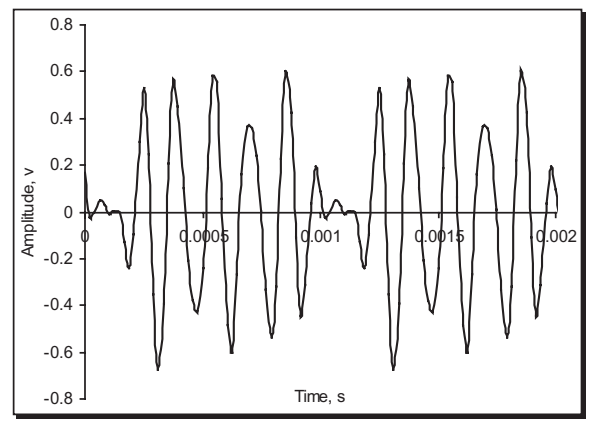

(a)

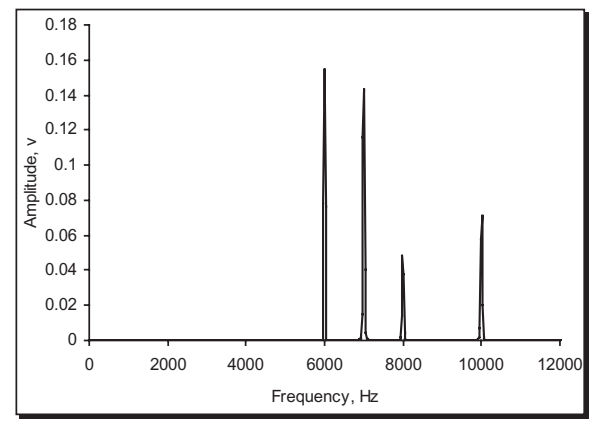

(b)

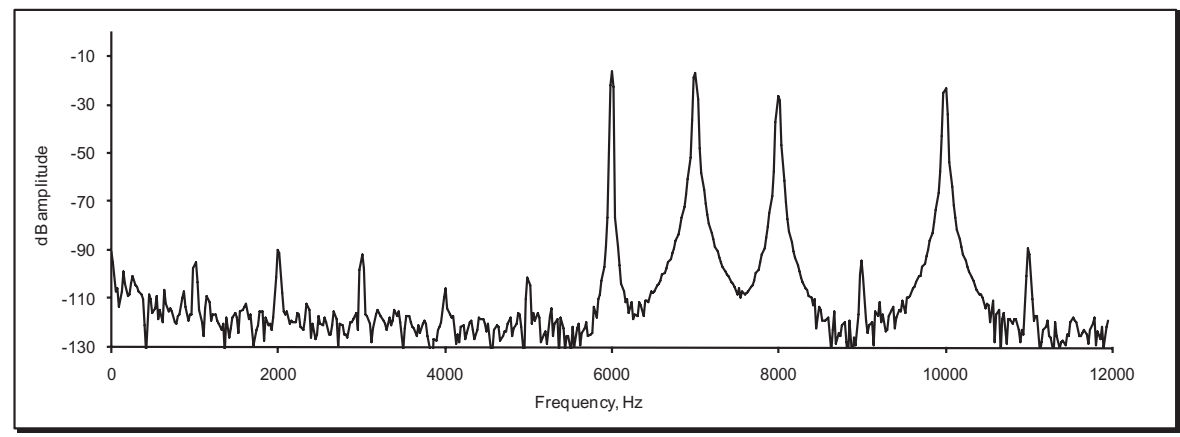

(c)

Fig. 6 (a) Composite waveform; (b) its spectrum, generated by the digital interpolation system; (c) spectrum in $d B$ scale.

of parallel interpolators in conjunction with phase, gain and final summation stages. The three main advantages of this technique are low THD, very precise frequency resolution and ease of real-time manipulation. Such properties are very useful in the performance characterisation and calibration of high-quality audio amplifiers, which traditionally are tested using signal injection equipment costing significantly more than this digital system. ${ }^{14}$ In addition, certain forms of magnetic field imaging for nondestructive testing rely on the use of low-noise signals art specific frequencies, and within this context this instrument represents a very convenient and economical excitation source. ${ }^{15}$

In teaching laboratories, digital waveform generators are being increasingly used at both an undergraduate and postgraduate level. They are significantly more flexible than their analogue counterparts, and students quickly appreciate the many advantages that they offer for circuit and project testing. However, unless students receive specific instruction on the manner of operation of digital function generators, it is difficult for them to understand the algorithmic engineering that represents the manner in which they operate. In the School of Electrical and Electronic Engineering at the University or Manchester, third-year undergraduate and Master's-level 
students receive formal instruction, through structured lectures, on digital waveform synthesis using both the look-up table approach and the method based on approximation through interpolation, described below. During laboratory sessions, they experience how the theoretical nature of the subject is realised at a practical level: students make use of the Signal Wizard Systems hardware ${ }^{\circledR}$ (Signal Wizard 2.5), which employs both of the above algorithms for function generation. Through a variety of experiments, students quickly gain an appreciation of the relative merits and limitations of each technique. Furthermore, THD comparisons with analogue methods establish the principle that the digital approach is inherently superior in terms of noise performance. Through this route, students quickly learn that the equations, described in some detail in the lectures (and elaborated below), have real and practical significance at an engineering level.

A typical digital interpolation system as described is superior to analogue function generators in several respects. First, the hardware cost is low, typically less than a quarter of the analogue equivalent. Second, the frequency resolution is absolute, governed only by the word length and the DAC clock rate. For example, a 24-bit system operating with a DAC clock rate of $48 \mathrm{kHz}$, the frequency resolution is $2.86 \mathrm{mHz}$; at a clock rate of $4 \mathrm{kHz}$ it is $0.238 \mathrm{mHz}$. It is important to stress that this value is constant over the entire operational range of the device. In contrast, an analogue function generator, which typically uses a continuously variable dial, will typically incorporate a frequency range selector, i.e. $0-10 \mathrm{~Hz}, 10-100 \mathrm{~Hz}$ etc, up to $10 \mathrm{kHz}-100 \mathrm{kHz}$ (for audio generators). Within each range, there will be a resolution of perhaps $1 \%$. There is moreover, a problem with drift and repeatability. The most significant advantage of the digital interpolation system is its ability to synthesise waveforms comprising many harmonics, each of which can be adjusted precisely in real-time respecting frequency, phase and gain, something of which neither an analogue system nor a system using a digital look-up table is capable.

In summary, the proposed system offers advantages over several existing methods of function generation, without incurring the attendant penalties. The look-up table is flexible with regard to arbitrary function generation, but is difficult to alter in real time and requires large memory resource for accurate function synthesis. In contrast, the sine-wave interpolation algorithm cannot generate arbitrary functions but has minimal memory requirement and can be altered in real time. Analogue function generators are also restricted in the range of functions they can produce and low-cost devices have modest THD performance. However, they can obviously be adjusted in real-time. This new system has the ability to synthesise arbitrary functions with very modest memory requirements, has good THD performance and, critically, each individual harmonic of a function can be adjusted (respecting frequency, amplitude and phase) during run-time.

\section{Conclusion}

A cost-effective, digital waveform synthesiser has been developed that employs a bank of parallel interpolators to evaluate a polynomial equation, which approximates, to a very high level of accuracy, a sine wave function. Total harmonic distor-

International Journal of Electrical Engineering Education, Volume 50, Number 3 (July 2013), (C Manchester University Press 
tion measurements have confirmed that the purity of the sine waves produced are significantly superior to those of a typical laboratory-type analogue function generator. The ability of the system to synthesise elaborate functions, combined with its capability for precise real-time control of frequency, phase and gain parameters, suggests that in many embedded and general purpose applications, the use of this system would offer significant benefits over more traditional approaches.

\section{References}

1 J. C. Humez,, 'Sine waves in programmable logic', Elektor Electronics, 35 (2009), 38-42.

2 L. Cordesses, 'Direct Digital Synthesis: A tool for periodic wave generation (Part 1)', IEEE Signal Proc. Mag., 21 (2004), 50-54.

3 L. Cordesses, 'Direct Digital Synthesis: A tool for periodic wave generation (Part 2)', IEEE Signal Proc. Mag., 21 (2004), 110-117.

4 Low Power $20 \mathrm{~mW} 2.3 \mathrm{~V}$ to $5.5 \mathrm{~V}$ Programmable Waveform Generator. Data Sheet, Analog Devices, Norwood, MA, USA (2003).

5 T. Abeyasekera, C. M. Johnson, D. J. Atkinson and M. Armstrong, 'Elimination of sub harmonics in direct look-up table (DLT) sine wave reference generators for low-cost microprocessor-controlled inverters', IEEE Trans. Power Electron., 18(6) (2003), 1315-1321.

6 A. Bateman and I. Paterson-Stephens, The DSP Handbook (Prentice Hall, Englewood Cliffs, NJ, 2002).

7 K. A. Stroud, Engineering Mathematics, 6th edn (Palgrave Macmillan, London, 2007).

8 A. Ashrafi, R. Adhami, L. Joiner and P. Kaveh, 2004. 'Arbitrary waveform DDFS utilizing Chebyshev polynomials interpolation', IEEE Trans. Circuits and Systems, 51 (2004),1468-1475.

9 R. G. Lyons, Streamlining Digital Signal Processing (Wiley, New York, 2007).

10 A. M. Sodagar, 'Mapping from phase to sine-amplitude in direct digital frequency synthesisers using parabolic approximation', IEEE Trans. Circuits Systems II: Analog and Digital Signal Processing, 47 (2000), 1452-1457.

11 J. A. Pineiro, S. F. Oberman, J. M. Muller and J. D. Bruguera, 'High-speed function approximation using a minimax quadratic interpolator', IEEE Trans. Comput., 54 (2005), 304-318.

12 A. Samarah, M. Al-Ibrahim and O. Loffeld, 'An efficient method for generating coherent digital sweep signals', in Proc. First International Conference on Computational Intelligence, Communication Systems and Networks (CICSYN), 23-25 July, Indore, India, 2009 (IEEE, Piscataway, NJ, 2009), pp.79-85.

13 Dai Xiang-ming, 'Implementation of arbitrary function generator (AFG) with linear interpolation', J. Electron Devices, 31 (2008), 1397-1400.

14 S. Maleczek, 'Optimisation of the total harmonic distortions of the acoustic vacuum tube push-pull amplifier', Archives of Acoustics, 33 (2008), 103-109.

15 P. Gaydecki, G. Miller, B.Fernandes and H. Zaid M. Hussin, 'Detection of magnetic fields highly attenuated by the skin effect through a ferrous steel boundary using a super narrowband digital filter, IEEE Trans. Instrument. Measurement, 57 (2008), 1171-1176. 